\title{
On degenerate multi-row Gomory cuts
}

\author{
Giacomo Zambelli*
}

April 2008

\begin{abstract}
Borozan and Cornuéjols introduced a semi-infinite relaxation for mixed-integer linear programs, derived from the simplex tableau relative to a fractional vertex $f$ of the linear relaxation. They showed that any minimal valid inequality for such relaxation is determined by a maximal lattice-free convex set containing $f$. In this note we show that, for the purpose of generating cuts for the original MIP, one needs to consider only maximal lattice-free convex bodies with $f$ in the interior.
\end{abstract}

Most cutting plane algorithms use cuts, such as Gomory's Mixed Integer Cuts, deduced by one single tableau inequality by arguments on the integrality and nonnegativity of some of the variables involved. The idea of studying inequalities that can be inferred by multiple rows of the simplex tableau has recently gained interest through the work of Dey and Richard [4], Dey et Al. [5], Andersen et Al. [1], Borozan and Cornuéjols [2], Cornuéjols and Margot [3], Dey and Wolsey [6], while Espinoza [7] reports promising computational results.

The following model, obtained from the simplex tableau by dropping the nonnegativity constraints on the basic variables and the integrality constraints on the non-basic ones, has been studied by Andersen et Al. [1] in the case of two tableau rows, and their results have been generalized by Borozan and Cornuéjols [2] to the case of multiple rows;

$$
\begin{aligned}
x & =f+\sum_{j=1}^{k} r^{j} s_{j}, \\
x & \in \mathbb{Z}^{q}, \\
s & \in \mathbb{R}_{+}^{k} .
\end{aligned}
$$

Here $f, r^{1}, \ldots, r^{k} \in \mathbb{Q}^{q}$ and $f \notin \mathbb{Z}^{q}$. Let $R_{f}\left(r^{1}, \ldots, r^{k}\right)$ be the convex hull of vectors $s \in$ $\mathbb{R}^{k}$ for which there exists $x \in \mathbb{R}^{q}$ such that $(x, s)$ satisfies (1). Since all data are rational,

*Dipartimento di Matematica Pura e Applicata, Universitá di Padova, Via Trieste 63, 35121 Padova, Italy. (giacomo@math.unipd.it) 
$R_{f}\left(r^{1}, \ldots, r^{k}\right)$ is a rational polyhedron. Since the recession cone of $R_{f}\left(r^{1}, \ldots, r^{k}\right)$ is $\mathbb{R}_{+}^{k}$, then any irredundant valid inequality for $R_{f}\left(r^{1}, \ldots, r^{k}\right)$ can be written in the form $\alpha^{\top} x \geq 1$ where $\alpha \in \mathbb{Q}_{+}^{k}$. Such inequality is said minimal if there is no valid inequality $\left(\alpha^{\prime}\right)^{\top} x \geq 1$ such that $\alpha^{\prime} \leq \alpha$ and $\alpha \neq \alpha^{\prime}$. Clearly irredundant inequalities are also minimal.

Borozan and Cornuéjols [2] proposed to study the following semi-infinite relaxation, related to Gomory and Johnson's infinite group problem [8],

$$
\begin{aligned}
& x=f+\sum_{r \in \mathbb{Q}^{q}} r s_{r}, \\
& x \in \mathbb{Z}^{q}, \\
& s \geq 0 \text { with finite support },
\end{aligned}
$$

where $s=\left(s_{r}\right)_{r \in \mathbb{Q}^{q}}$ is said to have finite support if it has a finite number of non-zero components. Let $R_{f}$ be the convex hull of all $s \in \mathbb{R}_{+}^{\mathbb{Q}^{q}}$ for which there exists $x \in \mathbb{R}^{q}$ such that $(x, s)$ satisfies $(2)$. Any valid inequality for $R_{f}$ is of the form

$$
\sum_{r \in \mathbb{Q}^{q}} \psi(r) s_{r} \geq 1
$$

where $\psi: \mathbb{Q}^{q} \rightarrow \mathbb{Q} \cup\{+\infty\}$. We say that such $\psi$ is a valid function; $\psi$ is said a minimal valid function if there is no valid function $\psi^{\prime}$ such that $\psi^{\prime}(r) \leq \psi(r)$ for all $r \in \mathbb{Q}^{q}$ and $\psi^{\prime}(r)<\psi(r)$ for at least one $r \in \mathbb{Q}^{q}$. Clearly we are only interested in minimal valid functions. Notice that any inequality valid for $R_{f}\left(r^{1}, \ldots, r^{k}\right)$ is of the form $\sum_{j=1}^{k} \psi\left(r^{j}\right) s_{j} \geq 1$ for some valid function $\psi$.

Borozan and Cornuéjols [2] show that, if $\psi$ is a minimal valid function, then $\psi$ is nonnegative, positively homogeneous (i.e. $\psi(\lambda r)=\lambda \psi(r)$ for all $\lambda \geq 0$ ), piece-wise linear, and convex. Since $\psi$ is a convex function defined in $\mathbb{Q}^{q}$, which is dense in $\mathbb{R}^{q}$, then there exists a unique convex function $\bar{\psi}$ defined on $\mathbb{R}^{q}$ that coincides with $\psi$ on $\mathbb{Q}^{q}$, so here for simplicity we just consider $\psi$ itself to be defined on $\mathbb{R}^{q}$. Borozan and Cornuéjols further show that, if $\psi$ is a minimal valid function, then the set

$$
B_{\psi}=\left\{x \in \mathbb{R}^{q} \mid \psi(x-f) \leq 1\right\}
$$

is a maximal lattice-free convex set, i.e. a maximal convex set with no point of $\mathbb{Z}^{q}$ in the interior. Clearly $f \in B_{\psi}$, because by positive homogeneity $\psi(f-f)=0$.

If $\psi$ is finite everywhere, then $\psi$ is continuous in $\mathbb{R}^{q}$, because every convex function is continuous in the interior of its domain. Thus, in this case, $B_{\psi}$ is closed and its boundary is $\left\{x \in \mathbb{Q}^{q} \mid \psi(x-f)=1\right\}$, hence $f$ is in the interior of $B_{\psi}$. Conversely, if $f$ is in the interior of $B_{\psi}$, then by positive homogeneity $\psi$ must be finite everywhere. We say that $\psi$ is degenerate if $f$ is on the boundary of $B_{\psi}$ (or equivalently if $\psi$ is not finite everywhere), while $\psi$ is nondegenerate if $f$ is in the interior of $B_{\psi}$. While a maximal lattice-free convex 
set $B$ with $f$ in the interior uniquely defines a valid function $\psi$ such that $B_{\psi}=B$ by positive homogeneity, in the case where $f$ is on the boundary such a $\psi$ is not uniquely determined by $B$, and in fact in order to define a degenerate valid function, one needs to define one maximal-lattice free convex set on each of the faces of $B$ containing $f[2]$. Furthermore, if $\psi$ is non-degenerate, then $\psi$ has at most $2^{q}$ linear pieces [2], whereas if $\psi$ is degenerate the bound on the number of pieces is exponentially larger.

The theorem we prove in this note implies that, from the practical point of view of generating cutting planes for the finite dimensional set $R_{f}\left(r^{1}, \ldots, r^{k}\right)$, one needs not be concerned with these complications arising from degenerate inequalities, because the only minimal inequalities arise from valid nondegenerate functions. This was shown by Cornuéjols and Margot [3] for the case that $q=2$ with an ad-hoc proof.

Theorem 1 Given a minimal valid inequality $\alpha^{\top} s \geq 1$ for $R_{f}\left(r^{1}, \ldots, r^{k}\right)$, there exists a nondegenerate minimal valid function $\psi$ such that $\psi\left(r^{j}\right)=\alpha_{j}$ for all $j=1, \ldots, k$.

Proof. We only need to show the existence of a finite valid function $\psi$ such that $\psi\left(r^{j}\right)=\alpha_{j}$ for $j=1, \ldots, k$. Define, for each $r \in \mathbb{R}^{q}, \tilde{\psi}(r)=\min \left\{\alpha^{\top} s \mid \sum_{j=1}^{k} r^{j} s_{j}=r, s \geq 0\right\}$, where $\tilde{\psi}(r)=+\infty$ if $r$ cannot be written as a conic combination of $r^{1}, \ldots, r^{k}$. Then $\tilde{\psi}$ is valid for $R_{f}$, albeit in general neither minimal nor finite, and $\tilde{\psi}\left(r^{j}\right)=\alpha_{j}$ for $j=1, \ldots, k$ because of the minimality of $\alpha^{\top} x \geq 1$. Clearly $\tilde{\psi}$ is homogeneous and convex, and $B_{\tilde{\psi}}=\left\{x \in \mathbb{R}^{q}: \exists s \geq 0\right.$ s.t. $\left.x=f+\sum_{j=1}^{k} r^{j} s_{j}, \alpha^{\top} s \leq 1\right\}$. Notice that $B_{\tilde{\psi}}$ is a rational polyhedron, since it is the projection onto the space $s_{j}=0, j=1, \ldots, k$, of a rational polyhedron in $\mathbb{R}^{q+k}$.

We observe that any face of $B_{\tilde{\psi}}$ containing $f$ does not contain an integral point in its relative interior. Suppose not, and let $F$ be a face of $B_{\tilde{\psi}}$ such that $f \in F$, and $\bar{x}$ be an integral point in the relative interior of $F$. Thus there exists $\lambda>1$ such that $f+\lambda(\bar{x}-f) \in F$. Since $\tilde{\psi}$ is positively homogeneous, $\tilde{\psi}(f-f)=0$, and $\tilde{\psi}(\bar{x}-f) \geq 1$, then $\tilde{\psi}(\lambda(\bar{x}-f))=\lambda \tilde{\psi}(\bar{x}-f)>1$, contradicting the fact that $f+\lambda(\bar{x}-f) \in B_{\tilde{\psi}}$.

Since $B_{\tilde{\psi}}$ is a polyhedron, $B_{\tilde{\psi}}=\{x \mid A x \leq b\}$ for some $m \times q$ matrix $A$ with rows $a^{1}, \ldots, a^{m}$, and vector $b \in \mathbb{R}^{m}$. Because $B_{\tilde{\psi}}$ is rational, we may assume that $(A, b)$ is integral. If $a^{i} f<b_{i}$ for all $i=1, \ldots, m$, then $f$ is in the interior of $B_{\tilde{\psi}}$ and we are done. So assume that $f$ satisfies at equality the first $h$ constraints of $A x \leq b$, and none of the other constraints. Let $b^{\prime}$ be the vector defined by $b_{i}^{\prime}=b_{i}+1, i=1, \ldots, h, b_{i}^{\prime}=b_{i}$, $i=h+1, \ldots, m$. Let $B=\left\{x \in \mathbb{Q}^{q} \mid A x \leq b^{\prime}\right\}$. Clearly $B \supset B_{\tilde{\psi}}$, and $f$ is in the interior of $B$ because $A f<b^{\prime}$.

We show that $B$ does not contain any integral point in its interior. Suppose not, and let $\bar{x}$ be an integral point satisfying $A \bar{x}<b^{\prime}$. Since $\bar{x}$ and $(A, b)$ are integral, and by definition of $b^{\prime}, \bar{x}$ satisfies $a^{i} \bar{x} \leq b_{i}, i=1, \ldots, h, a^{i} \bar{x}<b_{i}, i=h+1, \ldots, m$. Let $J$ be the set of indices $i$ such that $a^{i} \bar{x}=b_{i}$ (possibly $J=\emptyset$ ). Then $a^{i} x=b_{i}, i \in J$, define a face 
$F$ of $B_{\tilde{\psi}}$ (possibly $F=B_{\tilde{\psi}}$ ) that contains $f$ and such that $\bar{x}$ is in the relative interior of $F$, a contradiction.

For every $r \in \mathbb{Q}^{q}$, let $\lambda_{r}=\max \{\lambda \mid f+\lambda r \in B\}$. Notice that $\lambda_{r}>0$ for every $r \in \mathbb{Q}^{q}$ because $f$ is in the interior of $B$, and $\lambda_{r} \in \mathbb{Q}$ because $B$ is rational. Let the function $\psi: \mathbb{Q}^{q} \rightarrow \mathbb{Q}$ be defined by $\psi(r)=\lambda_{r}^{-1}$ for all $r \in \mathbb{Q}^{q}$. Then $B_{\psi}=B$ and, since $B$ does not contain any integral point in its interior, $\psi$ is valid for $R_{f}$. Because $B \supset B_{\tilde{\psi}}$, $\psi\left(r^{j}\right) \leq \tilde{\psi}\left(r^{j}\right)=\alpha_{j}$ for $j=1, \ldots, k$. By the minimality of $\alpha^{\top} x \geq 1, \psi\left(r^{j}\right)=\alpha_{j}$ for $j=1, \ldots, k$, thus $\psi$ satisfies the statement.

\section{References}

[1] K. Andersen, Q. Louveaux, R. Weismantel, L. Wolsey, Cutting Planes from Two Rows of the Simplex Tableau, Proceedings of IPCO XII, Ithaca, New York (2007).

[2] V. Borozan, G. Cornuéjols, Minimal Valid Inequalities for Integer Constraints, technical report (2007, revised August 2008).

[3] G. Cornuéjols, F. Margot, On the Facets of Mixed Integer Programs with Two Integer Variables and Two Constraints, Mathematical Programming A (2007), DOI 10.1007/s10107-008-0221-1.

[4] S.S. Dey, J.-P.P. Richard, Facets of Two-Dimensional Infinite Group Problems, Mathematics of Operations Research 33 (2008), 140-166.

[5] S.S. Dey, J.-P.P. Richard, Y. Li, L. A. Miller, Extreme inequalities for infinite group problems, 2006. http://www.optimization-online.org/DB_HTML/2006/ 04/1356.html.

[6] S.S. Dey, L.A. Wolsey, Lifting Integer Variables in Minimal Inequalities Corresponding to Lattice-Free Triangles. Proceedings of IPCO XIII (A. Lodi, A. Panconesi, G. Rinaldi Eds.), Bertinoro, Italy (2008), 463-475.

[7] D. Espinoza, Computing with multi-row Gomory Cuts, Proceedings of IPCO XIII (A. Lodi, A. Panconesi, G. Rinaldi Eds.), Bertinoro, Italy (2008), 214-224.

[8] R.E. Gomory, E.L. Johnson, Some continuous functions related to corner polyhedra, Mathematical Programming 3 (1972), 23-85. 